\title{
SNS HOM DAMPING REQUIREMENTS VIA BUNCH TRACKING*
}

\author{
R. Sundelin, Thomas Jefferson National Accelerator Facility, Newport News, VA 23606, USA \\ Dong-o Jeon, Sang-ho Kim, Marc Doleans, Oak Ridge National Laboratory, \\ Oak Ridge, TN 37831, USA
}

\begin{abstract}
Based on Higher Order Mode (HOM) field properties [1], the Spallation Neutron Source (SNS) HOM damping requirements have been determined by bunch tracking simulations. Transverse instabilities are found to be absent and transverse error magnifications are found to be acceptable if the loaded cavity Q for each mode is less than $10^{8}$ and the expected cavity-to-cavity frequency variation due to manufacturing tolerances is present. Longitudinal instabilities are absent if the loaded cavity Q for each HOM is less than $10^{8}$, the loaded cavity $\mathrm{Q}$ for each non-pi fundamental mode has the expected value, and the expected cavity-to-cavity frequency variation is present. Each of the two cavity types has three longitudinal modes that are close enough to a bunch spacing harmonic that one of the modes may occasionally get tuned onto the resonance; the power deposited as a function of $\mathrm{Q}$ has been calculated for this case. Deposited power [1] and transverse instabilities/magnifications [2] have also been determined analytically.
\end{abstract}

\section{INTRODUCTION}

The Spallation Neutron Source (SNS) will use two types of superconducting cavities to accelerate the $\mathrm{H}^{-}$ beam from $185 \mathrm{MeV}$ to $1000 \mathrm{MeV}$. 33 six-cell pi-mode elliptical cavities with a geometrical $\beta=0.61$, followed by 48 six-cell pi-mode elliptical cavities with a geometrical $\beta=0.81$ are used. The beam has macropulses at $60 \mathrm{~Hz}$, minipulses at $\sim 1 \mathrm{MHz}$ (introduced to provide an injection/extraction notch for the accumulator ring), and micropulses at $402.5 \mathrm{MHz}$. The purpose of the present study is to determine specific higher order mode (HOM) damping requirements for both betas in order to avoid instabilities or other unacceptable perturbations to the beam, and to deal in an acceptable fashion with HOM power generated. The study was performed by writing and using longitudinal and transverse tracking codes. These codes used predetermined HOM field properties [1] and known beam properties to determine cavitybeam interactions and HOM power generation for various damping conditions. The deviation from nominal of each bunch in transverse or longitudinal phase space is tracked as a function of bunch number (counting from the time the beam is turned on) and cavity number, as is the amplitude and phase of HOM excitation in each cavity as a function of number of bunches that have passed through that cavity.

\section{TRANSVERSE}

Since the SNS uses a single-pass linac (unlike typical superconducting electron linacs such as CEBAF), the possible cavity-beam instabilities are limited to cumulative and backward wave. Since the cavities are relatively short, and have no coupling to each other through external waveguides, backward wave instabilities were found not to be an issue [2]. Cumulative effects can be of two types: true instabilities, which can occur at almost any frequency, and error magnification [2], which is worst when an HOM frequency differs by of the order of 1 cavity bandwidth from a harmonic of a beam spectral line.

In both cases, the underlying physics is that a bunch which is off-center will induce a voltage in a deflecting mode of a cavity. This induced voltage will be vectorially added to any voltage already in the cavity due to previous bunches. The excitation of the cavity will also cause transverse deflection of any bunch passing through the cavity, with the magnitude of the deflection depending on the amplitude and phase of the cavity excitation, and on the particle momentum. The magnitude of this effect will tend to increase with time and with the distance downstream. Since the beam may not be centered in the cavity, the dipole components of quadrupole and sextupole modes for a beam $1 \mathrm{~cm}$ off axis (e.g., during tuning) have also been included in the study along with dipole modes. The reason that this type of instability can occur at almost any HOM frequency is that there is no predetermined betatron phase relationship between consecutive beam bunches. When a beam power spectral line falls on the edge of an HOM resonance, error magnification (rather than instability) occurs. Consecutive beam bunches, after the cavity reaches equilibrium, will encounter the same HOM amplitude and phase in a particular cavity. The bunches will simultaneously maintain this cavity excitation and be deflected by it.

An important limitation on the instability and position error amplification arises due the differences in HOM frequencies from cavity to cavity due to normal manufacturing tolerances. The magnitude of these errors was estimated based on an unpublished study by

* Sponsored by DE-AC05-00OR22725 
one of the authors for a nominally identical set of cavities made by the same manufacturing techniques as SNS cavities; that study found a standard deviation $\sigma=$ $0.00109 *\left|\mathrm{f}-\mathrm{f}_{\mathrm{o}}\right|$, where $\mathrm{f}$ is the HOM frequency and $\mathrm{f}_{\mathrm{o}}$ is the fundamental mode frequency (all values of $f_{o}$ were tuned to be the same). For the present study, 20\% of this $\sigma$ was used to allow for the possibility that the SNS cavities could have even smaller variations than the earlier set. Using this value of $\sigma$ yielded the result that there were no transverse instabilities for $\mathrm{Q}_{\mathrm{L}} \leq 10^{8}$, where $\mathrm{Q}_{\mathrm{L}}$ is the loaded quality factor. Note that this low a Q does not occur automatically (due to BCS losses) if $\mathrm{f}<14 * \mathrm{f}_{\mathrm{o}}$, so a modest amount of damping must be introduced.

In addition to the above cavity-to-cavity frequency spread, there is also an HOM frequency centroid error (relative to calculations) due to manufacturing tolerances; the above-referenced study found that \pm 0.00375 was the worst fractional deviation of the centroid. In this case, larger errors provide more opportunities for a beam spectral line to be "hit", so a \pm 0.008 fractional deviation was used as a conservative estimate for the SNS cavities. In the simulation, each HOM centroid frequency was shifted near to the largest beam power harmonic within this range. The largest resulting error magnification (relative to the output position error in the absence of HOMs) was $1 \pm$ 0.00062 , which is immeasurably different from unity.

\section{LONGITUDINAL INSTABILITIES}

Since the beam is not fully relativistic, longitudinal instabilities are also possible. In this case, a bunch energy error causes an arrival time error, which causes a change in phase of the HOM voltage induced. This change then causes a difference in the HOM voltage already in the cavity as seen by following bunches, which in turn causes a change in the energy error of those bunches. This effect, similar to the transverse case, can occur at almost any HOM frequency, since there is no predetermined phase relationship between consecutive bunch centroid oscillations about their equilibrium phase. For the longitudinal case, non-pi members of the fundamental passband, in addition to HOMs, can cause oscillations. For this passband, the $\mathrm{Q}_{\mathrm{L}}$ of each mode is determined by the field pattern of the mode and by the $\mathrm{Q}_{\mathrm{L}}$ of the fundamental mode, which is around $7 * 10^{5}$. The simulation uses the same manufacturing frequency spread algorithm as for the transverse case (which results in a particularly small spread for the $5 \pi / 6$ mode). An example of such an oscillation is shown in Figure 1, which shows bunch output energy vs. bunch number, using the expected $Q$ and frequency spread but artificially high $\mathrm{Z}^{*} \mathrm{~T}^{2} / \mathrm{Q}$ for the $5 \pi / 6$ mode. The result of the simulation was that instabilities and significant energy errors (caused by modes other than the fundamental mode) do not occur if the $\mathrm{HOM} \mathrm{Q}_{\mathrm{L}} \leq 10^{8}$.

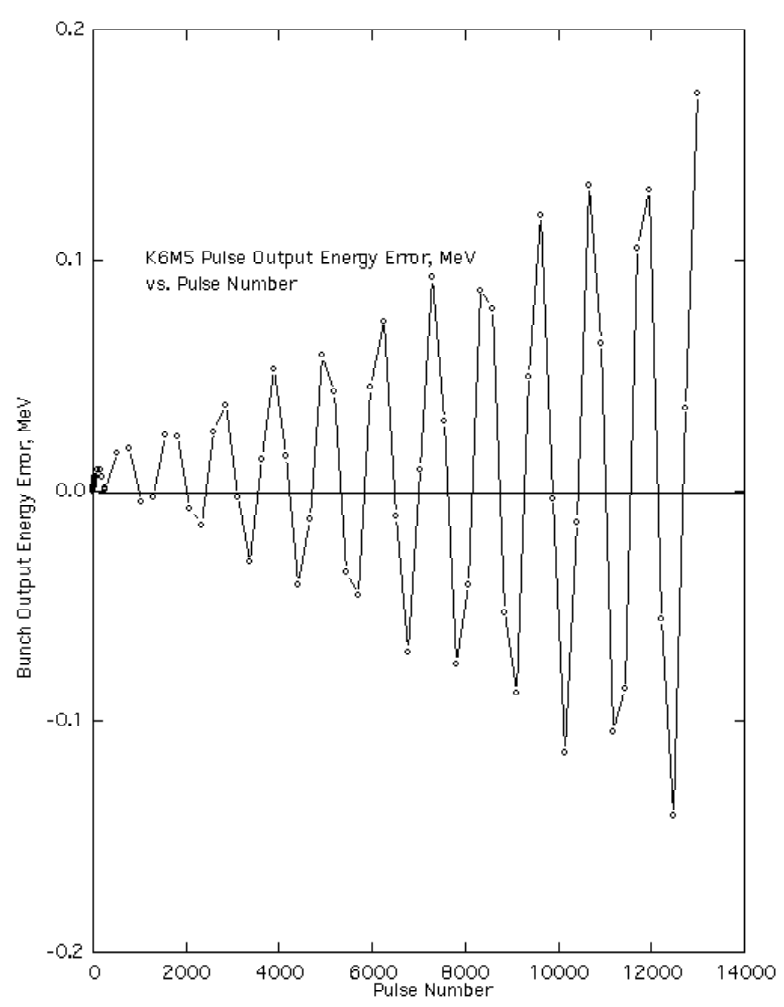

Figure 1: Bunch output energy error vs. bunch number, using the expected $\mathrm{Q}$ and frequency spread but artificially high $\mathrm{Z}^{*} \mathrm{~T}^{2} / \mathrm{Q}$ for the $5 \pi / 6$ mode.

\section{LONGITUDINAL HOM POWER}

The amount of HOM power that the beam can generate in a cavity depends on the HOM frequency, shunt impedance (which is a function of beam velocity), and $\mathrm{Q}_{\mathrm{L}}$ of the cavity. Where the power is deposited is determined by the sources of the damping. For this study, frequency centroids were artificially shifted to the highest beam power spectral line within the centroid error range. From the previously-mentioned study, the worst HOM frequency centroid fractional deviation (from the calculated value) was \pm 0.00375 . In case the SNS result is larger than this, \pm 0.008 was used for the SNS cavities. Non-pi members of the fundamental passband are treated differently, since tuning of the fundamental pi-mode tends to tune these modes. In this case, the earlier study yielded $\mid\left(\left(\mathrm{f}_{\text {meas }}-\mathrm{f}_{\text {calc }}\right) / \mathrm{f}_{\text {calc }}\right) /\left(\left(\mathrm{f}_{\text {calc }}{ }^{-}\right.\right.$ $\left.\left.\mathrm{f}_{\mathrm{o}}\right) / \mathrm{f}_{\mathrm{o}}\right) \mid \leq 0.027$, where $\mathrm{f}_{\text {meas }}$ is the measured frequency of a mode, and $\mathrm{f}_{\text {calc }}$ is its calculated frequency. For SNS, 2.5 times this limit was used as a conservative estimate. The next step in the simulation was to introduce a random frequency spread using the previously described value of $\sigma$. The cavity with the smallest random frequency shift then had its shift reset to zero.

The probability that any cavity frequency will fall on a significant beam power spectral line at any instant in time is very small. However, many factors can cause HOM frequencies to move around with time. One of these factors is intentional detuning of a cavity, using the mechanical tuner, when a cavity is to be taken out of 
service for any reason (e.g., its klystron failed); the "parking" frequency is different every time this is done. If a cavity is warmed up to degas the surface, the HOM frequencies will be different when it is cooled down. Changes in the liquid helium level and liquid helium pressure will have $f_{o}$ compensated by the mechanical tuner, but the HOM frequencies will all be different. Changes in the fundamental voltage, beam bunch phase, beam bunch charge, or beam velocity profile along the linac will require changes in the fundamental detuning angle, which will change all the HOM frequencies. Changes in the linac output energy will change the minibunch frequency, which will change many beam spectral line frequencies. During the 40 year life of a SNS cavity, the probability that it will have a HOM land on an important beam spectral line at least once approaches unity. To have a reliable accelerator, it is imperative that such an event destroy neither the beam properties nor the cavity.

Three HOMs were found in each cavity shape that have the potential to land on an important beam spectral line. The amount of power generated depends on $\mathrm{Q}_{\mathrm{L}}$. For the worst of these modes, $473 \mathrm{~W}$ would be generated at $\mathrm{Q}_{\mathrm{L}}=10^{8}$, falling to $1.34 \mathrm{~W}$ at $\mathrm{Q}_{\mathrm{L}}=10^{3}$. This result is shown in Figure 2.

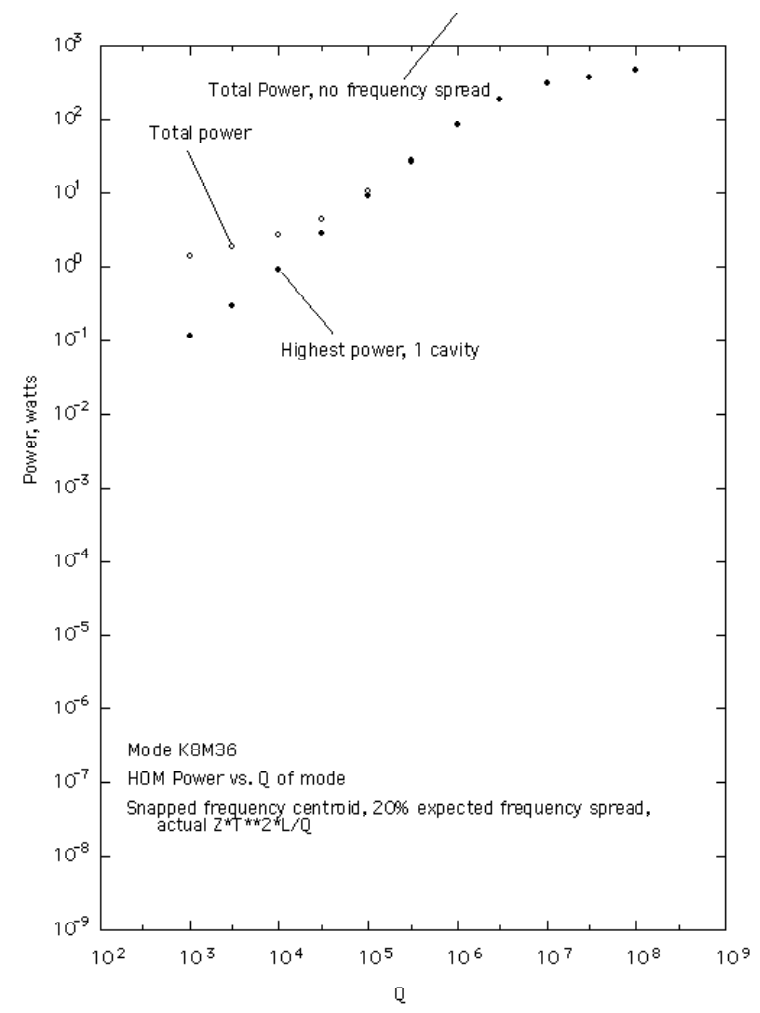

Figure 2. Average power generated vs. $\mathrm{Q}_{\mathrm{L}}$ for one $\beta$ $=0.81$ cavity having longitudinal mode 36 centered on a bunch spacing harmonic (solid circles), and for the entire ensemble of cavities where all other cavities differ from this frequency due to manufacturing tolerances (open circles).
The achieved damping will determine whether this power can be dissipated at $2.1 \mathrm{~K}$, or if it needs to be dissipated at the shield temperature, or even at room temperature.

\section{CONCLUSIONS}

Both transverse and longitudinal instabilities are avoided by relatively modest damping $\left(\mathrm{Q}_{\mathrm{L}} \leq 10^{8}\right.$ for HOMs, actual Qs for non-pi fundamental modes) and by $\geq 20 \%$ of nominal cavity-to-cavity frequency spread due to manufacturing tolerances.

The same criteria limit error multiplication near bunch spacing harmonics to $1 \pm 0.00062$, which is immeasurably different from unity.

To avoid probable destruction of the beam and/or cavity during the life of the accelerator, controlled damping of up to $473 \mathrm{~W}$ of HOM power generated in a cavity is needed.

\section{REFERENCES}

[1] Sang-ho Kim, Marc Doleans, Dong-o Jeon, and Ronald Sundelin, paper MPPH149, this conference.

[2] Dong-o Jeon, Jie Wei, Jean Delayen, Geoffrey Krafft, Ronald Sundelin, and Byung Yunn, paper TPPH313, this conference. 